Published by Al-Nahrain College of Medicine P-ISSN 1681-6579

E-ISSN 2224-4719

Email: iraqijms@colmed-alnahrain.edu.iq

http://www.colmed-alnahrain.edu.iq

http://www.iraqijms.net

Iraqi JMS 2018; Vol. 16(1)

\title{
NLRP3 Inflammasome: A Promising Theranostic Target in Inflammatory Diseases
}

\author{
Haider F. Ghazi PhD Microbiology \& Immunology \\ Dept. of Microbiology, College of Medicine, Al-Nahrain University, Baghdad, Iraq
}

\begin{abstract}
Currently, the evaluation and treatment of chronic inflammatory diseases relies on specific clinical signs accompanied with single or multiple marker(s) for that disease. Despite that, these diseases share a common innate immune mechanism involved upregulation of "Inflammasome" that have been proved in its implication in the pathologic mechanism (s) and direct irreversible conversion of pro-interleukin $1 \beta$ and pro-interleukin 18 into active $\mathrm{IL}-1 \mathrm{~b}$ and active IL-18 contributing in disease progression and tissue damage, suggesting a common theranostic target to reduce inflammatory mechanism.
\end{abstract}

Keywords NLRP3, inflammasome, theranostic marker

Citation Ghazi HF. NLRP3 Inflammasome: a promising theranostic target in inflammatory diseases. Iraqi JMS. 2018; Vol. 16(1): 1-3. doi: 10.22578/IJMS.16.1.1

List of abbreviation: PAMP: Pathogen associated molecular patterns; DAMP: Damage associated molecular pattern; NLRP3: Nucleotide binding oligomerization domain-like protein 3; ASC: Apoptosis-associated speck-like protein; TLR: toll like receptor; NF-kB: Nuclear factor kappa B.

$\mathrm{T}$ Theranostics is a term refers to examination of a biomarker molecule as a target for both diagnostic and therapeutic purposes allowing integration between in vitro diagnostics and reducing delay of treatment for optimum patients care (1). The main concept of immune system is defending against pathogen associated molecular pattern (PAMPs) as external pathogens and damage associated molecular pattern (DAMPs) as internal threats like uric acid and heat shock proteins mediated by certain pattern recognition receptors (PRRs) whom responsible for generation of inflammation and other effector mechanisms in diverse conditions ${ }^{(2)}$. The Nucleotide-binding oligomerization domain-like protein 3 (NLRP3) is the best characterized molecule belongs to a complex called "Inflammasome" in addition to apoptosis-associated speck-like protein (ASC) and procaspase- 1 resulting in irreversible immune activity through both proinflammatory cytokines (IL1 $\beta$ and IL18) ${ }^{(3,4)}$. NLRP3 inflammasome presents in inflammatory immune cells like: dendritic cells, macrophages and monocytes ${ }^{(5)}$. The activation process of the NLRP3 inflammasome appears to occur in two steps involves a priming or initiating signal in which, many PAMPs or DAMPs are recognized by toll like receptors (TLRs), leading to activation of nuclear factor kappa B (NF-kB)-mediated transcription of inflammasome proteins, including inactive NLRP3, prolL-1 $\beta$, and prolL- $18{ }^{(5)}$. Followed by the oligomerization of NLRP3 and subsequent assembly of NLRP3, ASC, and procaspase-1 into inflammasome complex allowing its autocleavage and activation. The activated caspase-1 enzyme in turn cleaves upregulated 
inactive proinflammatory cytokines: IL-1 and IL18 in to active IL-1 $\beta$ and IL-18 and secreting them into extracellular compartment resulting in induction of proinflammatory and proapoptotic pathways depending on type of threatening patterns ${ }^{(5,6)}$. In fact, the assembly of NLRP3 inflammasome and its activation happens downstream to many stimuli induced by various PAMPs or DAMPs showing its importance in playing an essential role various disease processes ${ }^{(7)}$.

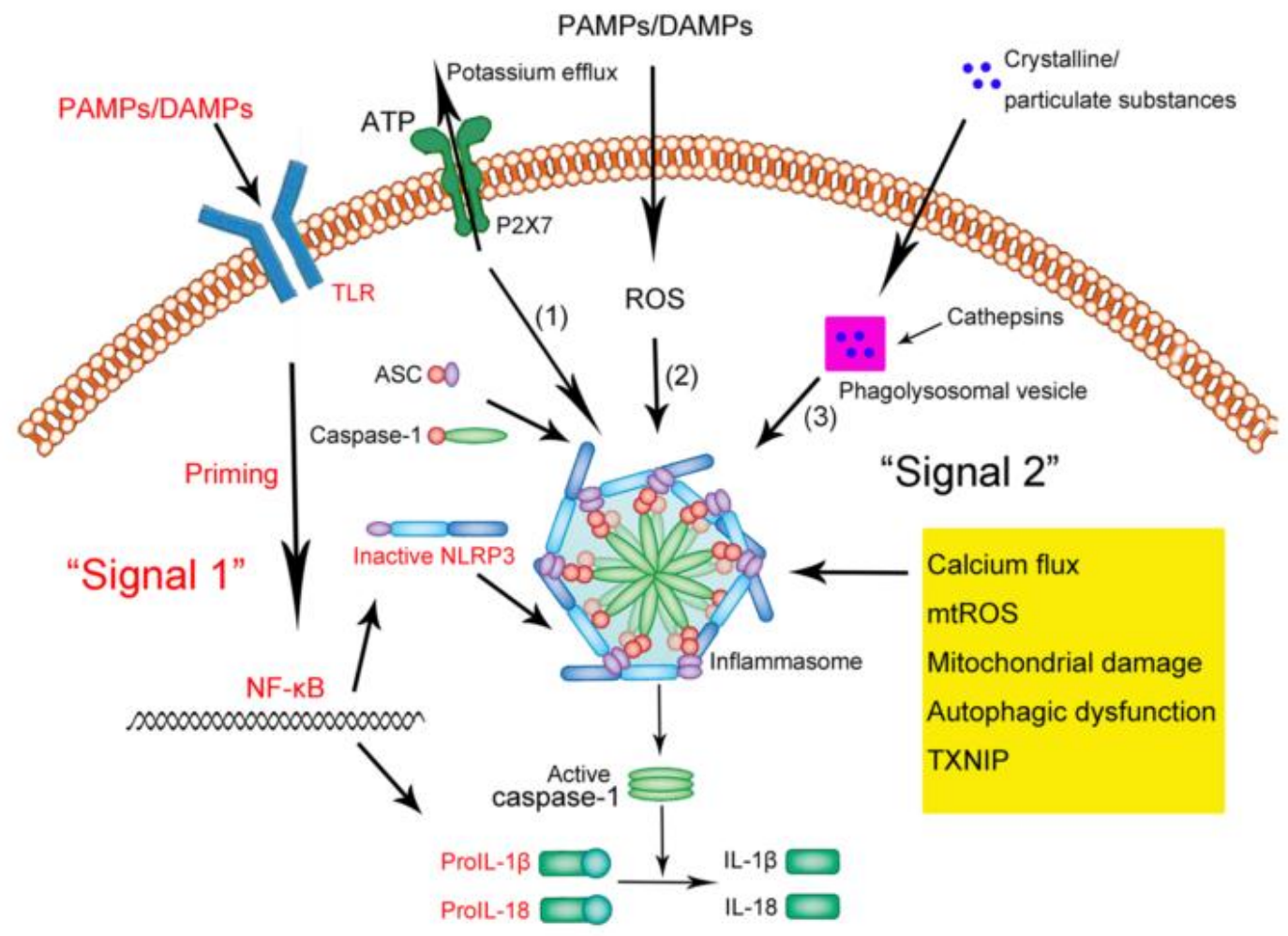

Figure 1: Illustration of the NLRP3 inflammasome activation (8)

The inappropriate activation of NLRP3 inflammasome can contribute in the initiation and progression of various diseases like: metabolic disorder and metabolic syndrome (9), atherosclerotic plaque in both atherosclerotic patients and animal model (10,11), NLRP3 inflammasome proteins up-regulated in patients with type 1 diabetes (12), IgA nephropathy (13), multiple sclerosis ${ }^{(14)}$ cancer (15) and other autoimmune diseases.

Consistent with the associations of NLRP3 inflammasome activation in these diseases, inhibiting the NLRP3 inflammasome may reduce their pathogenesis $(3,8,16)$. By another mean, targeting NLRP3 inflammasome will affect downstream signals preventing secretions of active proinflammatory processes. As well as an anti-infective ${ }^{(16)}$. Until recently, researchers found a selective direct inhibitor MCC950 specifically inhibited activation of NLRP3 that later on Novartis company funded Inflazome as a trade mark for this molecule ${ }^{(17)}$, (CY-09) ${ }^{(18)}$ and telmisartan (19).

In conclusion, all these evidences rising the suggestion for targeting NLRP3 inflammasome as a theranostic marker for inflammatory disease. Its suggested as a specific and sensitive biomarker superior than CRP, or other proinflammatory markers because its expressed downstream to real threat recognition by innate immune receptors and 
provide upstream signaling for activation and production of active proinflammatory cytokines (IL-1B and IL18) mediating inflammation and apoptotic signaling. In another hand, NLRP3 inflammasome as a valid target for treating inflammatory diseases.

\section{References}

1. Chen $X$, Wong S. Cancer Theranostics. Elsevier Science; 2014. eBook ISBN: 9780124078840.

2. Iwasaki A, Medzhitov R. Control of adaptive immunity by the innate immune system. Nat Immunol. 2015; 16(4): 343-53. doi: 10.1038/ni.3123.

3. Cook GP, Savic S, Wittmann $M$, et al. The NLRP3 inflammasome, a target for therapy in diverse disease states. Eur J Immunol. 2010; 40(3): 631-4. doi: 10.1002/eji.200940162.

4. He Y, Hara H, Nuez G. Mechanism and Regulation of NLRP3 Inflammasome Activation. Trends Biochem Sci. 2016; 41(12): 1012-21. doi: 10.1016/j.tibs.2016.09.002.

5. Guarda G, Zenger M, Yazdi AS, et al. Differential Expression of NLRP3 among Hematopoietic Cells. J Immunol. 2011; 186(4): 2529-34. doi: 10.4049/jimmunol.1002720.

6. Larock CN, Todd J, Larock DL, et al. IL-1 b is an innate immune sensor of microbial proteolysis. Sci Immunol. 2016; 1(2): eaah3539. doi: 10.1126/sciimmunol.aah3539.

7. Guo H, Callaway JB, Ting JP. Inflammasomes: mechanism of action, role in disease, and therapeutics. Nat Med. 2015; 21(7): 677-87. doi: 10.1038/nm.3893.

8. Shao $B Z, X u Z Q, H a n B Z$, et al. NLRP3 inflammasome and its inhibitors: a review. Front Pharmacol. 2015; 6: 262. doi: 10.3389/fphar.2015.00262.

9. Fulop $T$, Larbi A, Dupuis $G$, et al. Immunosenescence and inflamm-aging as two sides of the same coin: friends or foes? Front Immunol. 2018; 8: 1960. doi: 10.3389/fimmu.2017.01960.

10. Altaf $A, Q u P$, Zhao $Y$, et al. NLRP3 inflammasome in peripheral blood monocytes of acute coronary syndrome patients and its relationship with statins.
Coron Artery Dis. 2015; 26(5): 409-21. doi: 10.1097/MCA.0000000000000255.

11. Peng $K$, Liu $L$, Wei $D$, et al. $P 2 X 7 R$ is involved in the progression of atherosclerosis by promoting NLRP3 inflammasome activation. Int J Mol Med. 2015; 35(5): 1179-88. doi: 10.3892/ijmm.2015.2129.

12. Carlos D, Costa FRC, Leite JA, et al. NLRP3 inflammasome: from pathogenesis to therapeutic strategies in type 1 diabetes NLRP3 inflammasome: from pathogenesis to therapeutic strategies in type 1 diabetes. J Autoimm Disord. 2017; 3(2:33): 1-4.

13. Tsai Y-L, Hua K-F, Chen $A$, et al. NLRP3 inflammasome: Pathogenic role and potential therapeutic target for IgA nephropathy. Sci Rep. 2017; 7: 41123. doi: 10.1038/srep41123.

14. Keane RW, Dietrich WD, de Rivero Vaccari JP. Inflammasome proteins as biomarkers of multiple sclerosis. Front Neurol. 2018; 9: 135 . doi: 10.3389/fneur.2018.00135.

15. Thi HTH, Hong S. Inflammasome as a Therapeutic Target for Cancer Prevention and Treatment. J Cancer Prev. 2017; 22(2): 62-73. doi: 10.15430/JCP.2017.22.2.62.

16. Thacker JD, Balin BJ, Appelt DM, et al. NLRP3 inflammasome is a target for development of broadspectrum anti-infective drugs. Antimicrob Agents Chemother. 2012; 56(4): 1921-30. doi: 10.1128/AAC.06372-11.

17. Coll RC, Robertson AAB, Chae JJ, et al. A smallmolecule inhibitor of the NLRP3 inflammasome for the treatment of inflammatory diseases. Nat Med. 2015; 21(3): 248-55. doi: 10.1038/nm.3806.

18. Jiang $H$, He $H$, Chen $Y$, et al. Identification of a selective and direct NLRP3 inhibitor to treat inflammatory disorders. J Exp Med. 2017; 214(11): 3219-38. doi: 10.1084/jem.20171419.

19. Wei $X$, Hu CC, Zhang YL, et al. Telmisartan reduced cerebral edema by inhibiting NLRP3 inflammasome in mice with cold brain injury. J Huazhong Univ Sci Technolog Med Sci. 2016; 36(4): 576-83. doi: 10.1007/s11596-016-1628-1.

\section{E-mail: dr.haider.ghazi@colmed- alnahrain.edu.iq}

